\title{
Antagonistic Regulation of PAF1C and p-TEFb Is Required for Oligodendrocyte Differentiation
}

\author{
Suhyun Kim, ${ }^{\star}$ Jun-Dae Kim, ${ }^{2 \star}$ Ah-Young Chung, ${ }^{1}$ Hyung-Seok Kim, ${ }^{2}$ Young-Seop Kim, ${ }^{2}$ Myoung-Jin Kim, ${ }^{2}$ \\ Soonil Koun, ${ }^{2}$ You Mie Lee, ${ }^{2}$ Myungchull Rhee, ${ }^{3}$ Hae-Chul Park, ${ }^{1}$ and Tae-Lin Huh ${ }^{2,4}$ \\ ${ }^{1}$ Graduate School of Medicine, Korea University, Ansan, Gyeonggido 425-707, Republic of Korea, ${ }^{2}$ School of Life Science and Biotechnology, Kyungpook \\ National University, Daegu 702-701, Republic of Korea, ${ }^{3}$ Department of Biological Sciences, College of Biological Sciences and Biotechnology, Chungnam \\ National University, Daejeon 305-764, Republic of Korea, and ${ }^{4}$ Korea Basic Science Institute Daegu Center, Daegu 702-701, Republic of Korea
}

Oligodendrocytes are myelinating glial cells in the CNS and are essential for proper neuronal function. During development, oligodendrocyte progenitor cells (OPCs) are specified from the motor neuron precursor domain of the ventral spinal cord and differentiate into myelinating oligodendrocytes after migration to the white matter of the neural tube. Cell cycle control of OPCs influences the balance between immature OPCs and myelinating oligodendrocytes, but the precise mechanism regulating the differentiation of OPCs into myelinating oligodendrocytes is unclear. To understand the mechanisms underlying oligodendrocyte differentiation, an $N$-ethyl- $N$ nitrosourea-based mutagenesis screen was performed and a zebrafish leo1 mutant, dalmuri $\left(\right.$ dal $^{k n u \sigma}$ ) was identified in the current study. Leo1 is a component of the evolutionarily conserved RNA polymerase II-associated factor 1 complex (PAF1C), which is a positive regulator of transcription elongation. The $\mathrm{dal}^{k n u 6}$ mutant embryos specified motor neurons and OPCs normally, and at the appropriate time, but OPCs subsequently failed to differentiate into myelinating oligodendrocytes and were eliminated by apoptosis. A loss-offunction study of $c d c 73$, another member of PAF1C, showed the same phenotype in the CNS, indicating that PAF1C function is required for oligodendrocyte differentiation. Interestingly, inhibition of positive transcription elongation factor $b$ ( $p$-TEFb), rescued downregulated gene expression and impaired oligodendrocyte differentiation in the dal ${ }^{k n u}{ }^{k}$ mutant and Cdc73-deficient embryos. Together, these results indicate that antagonistic regulation of gene expression by PAF1C and p-TEFb plays a crucial role in oligodendrocyte development in the CNS.

\section{Introduction}

Oligodendrocytes are glial cells which myelinate axons in the CNS. In the developing neural tube, oligodendrocyte progenitor cells (OPCs) are generated from the ventral motor neuron precursor (pMN) domain after motor neurons are produced. After birth, OPCs exist as proliferative progenitors and differentiate into myelinating oligodendrocytes after migration to the white matter of the neural tube (Park et al., 2002; Rowitch, 2004). How-

Received Oct. 23, 2011; revised March 5, 2012; accepted April 1, 2012.

Author contributions: S. Kim, J.-D.K., H.-C.P., and T.-L.H. designed research; S. Kim, J.-D.K., A.-Y.C., H.-S.K., Y.-S.K., M.-J.K., and S. Koun performed research; Y.M.L. and M.R. analyzed data; S. Kim, J.-D.K., H.-C.P., and T.-L.H. wrote the paper.

This research was supported by the Brain Research Program (2011-0019233 for H.-C.P.) and Basic Science Research Program (2011-0026208 for T.-L.H.) through the National Research Foundation of Korea, funded by the Ministry of Education, Science and Technology. We thank W. Talbot for the generous gift of an anti-MBP antibody.

*S. Kim and J.-D.K. contributed equally to this work.

The authors declare no competing financial interests.

Correspondence should be addressed to either of the following: Hae-Chul Park, Graduate School of Medicine, Korea University, Ansan, Gyeonggido 425-707, Republic of Korea, E-mail: hcpark67@korea.ac.kr, or Tae-Lin Huh, School of Life Science and Biotechnology, Kyungpook National University, Daegu 702-701, Republic of Korea, E-mail: thuh@knu.ac.kr.

J.-D. Kim's present address: Department of Internal Medicine, Section of Cardiovascular Medicine, Yale School of Medicine, Int Med-Cardiology, P0 Box 208017, New Haven, CT 06520-8017.

H.-S. Kim's present address: Department of Neurobiology and Anatomy, University of Utah School of Medicine, Salt Lake City, Utah 84132-3401.

DOI:10.1523/JNEUROSCI.5344-11.2012

Copyright $\odot 2012$ the authors $\quad 0270-6474 / 12 / 328201-07 \$ 15.00 / 0$ ever, the precise mechanisms that regulate the differentiation of OPCs into myelinating oligodendrocytes are unclear.

Eukaryotic transcription begins with the assembly of a preinitiation complex and recruitment of RNA polymerase II (Pol II) to the promoter, followed by initiation, elongation and termination of RNA synthesis. In many cases, recruitment of Pol II to the promoter is necessary and sufficient for the activation of gene transcription. However, recent genome-wide studies have revealed the existence of the promoter-proximal pausing of Pol II for the expression of numerous genes that respond to specific stimuli and developmental signals (Muse et al., 2007; Zeitlinger et al., 2007; Core et al., 2008). In the promoter-proximal pausing model, transcription elongation factor, DRB sensitivity-inducing factor (DSIF), collaborates with negative elongation factor (NELF) to inhibit Pol II-mediated elongation shortly after transcription initiation, and positive transcription elongation factor $b$ ( $\mathrm{p}$-TEFb) is required to release paused Pol II (Price, 2008; Chiba et al., 2010). Pol II-associated factor 1 complex (PAF1C) is a positive regulator of transcription elongation and is commonly composed of five components: Paf1, Rtf1, Cdc73, Ctr9, and Leo1 (Hager et al., 2009; Jaehning, 2010). Interestingly, a recent study has shown that antagonistic regulation by $\mathrm{p}-\mathrm{TEFb}$ and PAF1C is required for the relief of Pol II pausing in erythropoiesis (Bai et al., 2010).

In the current study, we describe the function of PAF1C in the regulation of oligodendrocyte development using the ze- 

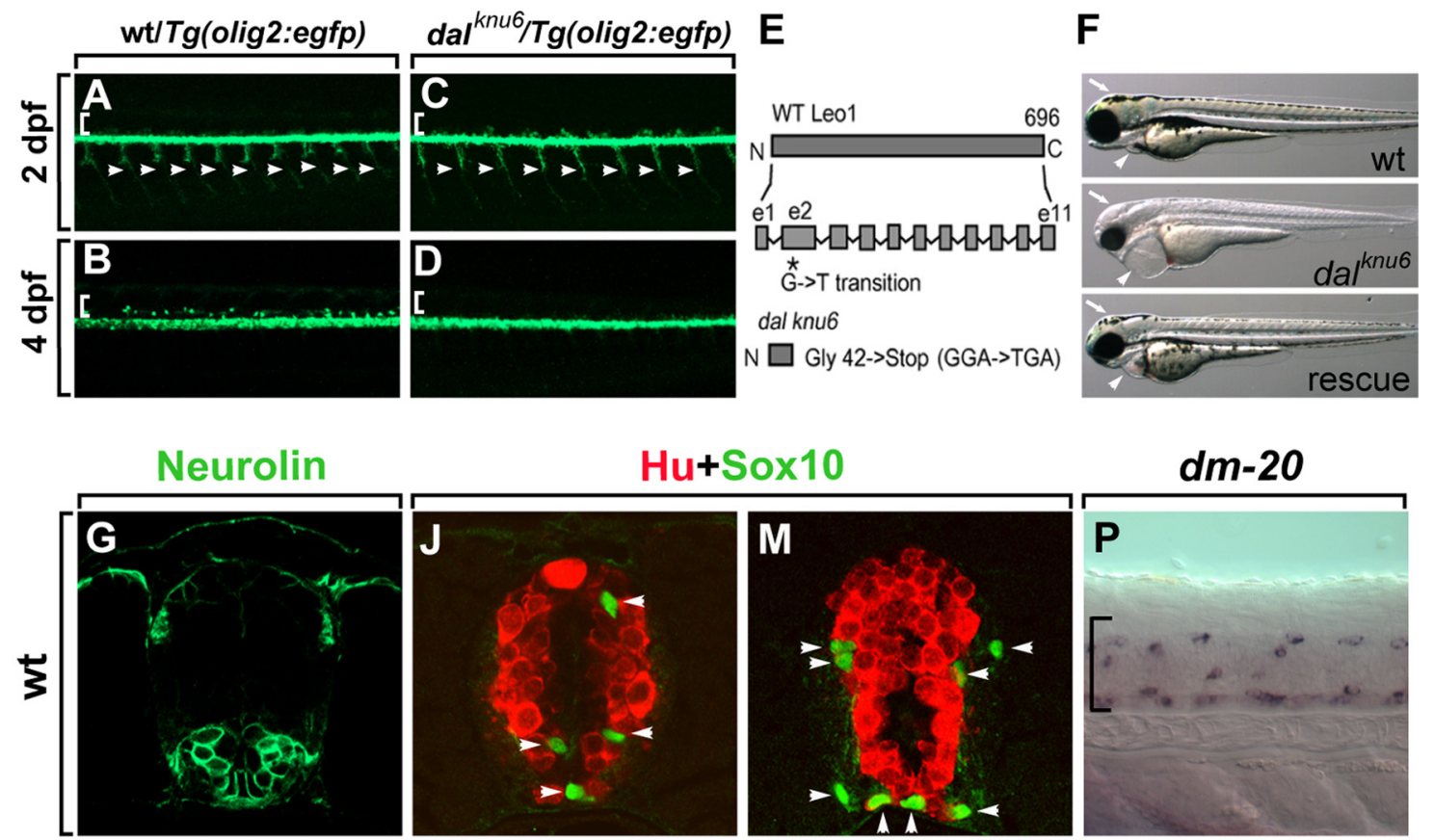

Hu+Sox10
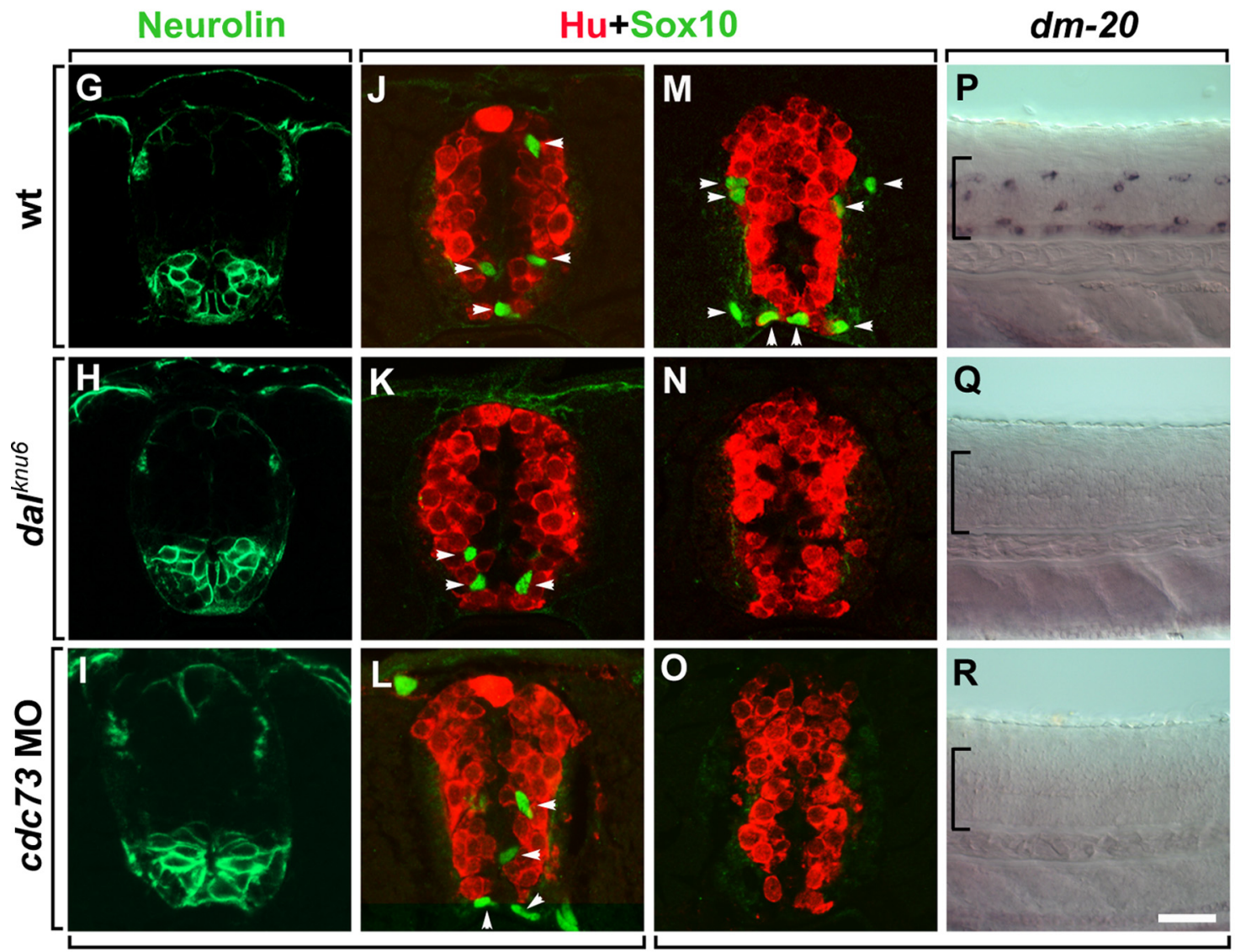

$2 \mathrm{dpf}$

$4 \mathrm{dpf}$

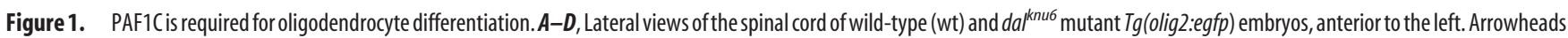
indicate motor axon bundles and bracketed areas indicate dorsal spinal cord. $\boldsymbol{E}$, Schematic representation of wt and mutant Le01. $F$, Phenotypes of wt and dal ${ }^{k n u}$ mutant embryos at $4 \mathrm{dpf}$. Arrows indicate pigment cells in the brain and arrowheads indicate heart. The dal ${ }^{k n u}$ mutation was rescued by injection of WT leo $1 \mathrm{mRNA}$. G-O, Transverse sections of spinal cord of wt $(\mathbf{G}, \mathbf{J}, \boldsymbol{M})$, daknuG mutant $(\boldsymbol{H}, \boldsymbol{K}, \boldsymbol{N})$ and $c d c 73 \mathrm{M} 0$-injected embryos $(\boldsymbol{I}, \mathbf{L}, \mathbf{0})$, orientated with dorsal to the top. Antibodies and stages are indicated on each panel. $\boldsymbol{P}-\boldsymbol{R}$, Lateral views of the spinal cord of wt $(\boldsymbol{P})$, dak ${ }^{k n u} \mathbf{6}$ mutant $(\mathbf{Q})$ and $c d c 73 \mathrm{MO}$-injected embryos $(\boldsymbol{R})$ hybridized with a $d m$-20RNA probe, orientated with anterior to the left. Bracketed area indicates spinal cord. Scale bars: $\boldsymbol{A}-\boldsymbol{D}, 80 \mu \mathrm{m} ; \mathbf{G}-\boldsymbol{R}, 20 \mu \mathrm{m}$.

brafish dalmuri $\left(\right.$ dal $\left.{ }^{k n u 6}\right)$ mutant, which has a mutation in Leo1 (a component of the PAF1C), and a $c d c 73$ morphant. The $d a l^{k n u 6}$ mutant embryos and $c d c 73$ morphants specified OPCs normally, and at the appropriate time, in the CNS but OPCs subsequently failed to differentiate into myelinating oligodendrocytes and were eliminated by apoptosis, indicating that PAF1C function is required for oligodendrocyte differentiation. Interestingly, inhibition of $\mathrm{p}-\mathrm{TEFb}$ function rescued downregulated gene expression and impaired oligodendrocyte differentiation in $d a l^{k n u 6}$ mutant embryos and $c d c 73$ morphants, indicating that functional antagonism between PAF1C and p-TEFb plays a crucial role in regulating oligodendrocyte development.

\section{Materials and Methods}

Fish lines. Wild-type AB, $\operatorname{Tg}$ (olig2:egfp) (Shin et al., 2003), $\operatorname{Tg}(n k x 2.2 a$ : megfp;olig2:dsred) (Kucenas et al., 2008) and dalmuri ${ }^{\text {knu6 }}$ (dal $\left.{ }^{k n u 6}\right)$ zebrafish mutant of either sex were used for this study.

Morpholino injection and rescue experiments. For knock-down of Leo1 and Cdc73, leo1 exon1 splicing blocking morpholinos (MOs; leo1 MO), $c d c 73$ exon 2 splicing blocking MOs $(c d c 73 \mathrm{MO})$ were purchased from Gene-Tools: leo1 MO, 5'-TATGAATGTACCTCGTTGCTCATTG-3'; $c d c 73 \mathrm{MO}, 5^{\prime}$-TTACTTACTGCAGCTCTCCGCACAT-3'. The specificity of $l e o 1$ and $c d c 73 \mathrm{MO}$ were verified by Western blot analysis with anti-Leol antibody (ab33157, Abcam) and reverse transcription PCR (RT-PCR), respectively (data not shown). For rescue experiments, leo1 mRNA (150 pg) were injected into one-cell embryos. 


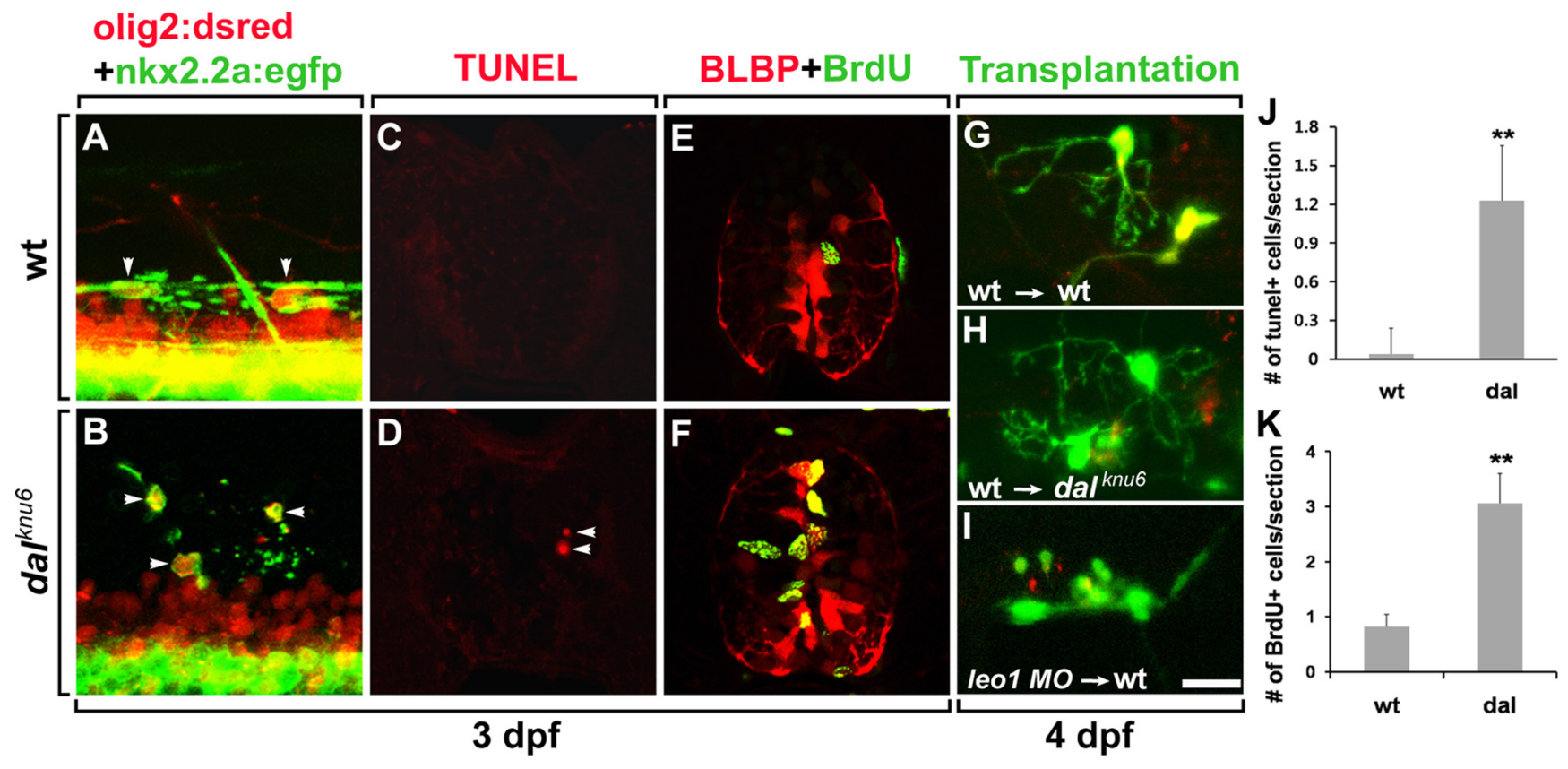

Figure 2. Cell autonomous functioning of Le01 is required for the survival and differentiation of OPCs. $\boldsymbol{A}, \boldsymbol{B}$, Lateral views of wt $(\boldsymbol{A})$ and dalknu $\mathrm{mutant}(\boldsymbol{B}) \operatorname{Tg}(n k \times 2.2 a: m e g f p:: 0 l i g 2: d s r e d)$ spinal cord, orientated with anterior to the left. Arrowheads indicate olig2 ${ }^{+} / \mathrm{nkx2.2a^{+ }}$ myelinating oligodendrocytes. $\mathbf{C}-\boldsymbol{F}$, Transverse sections of spinal cord, orientated with dorsal to the top. TUNEL staining of wt $(\boldsymbol{C})$ and dal ${ }^{k n u}$ mutant $(\boldsymbol{D})$ embryos. Arrowheads indicate TUNEL ${ }^{+}$cells. BrdU treatment and labeling of wt $(\boldsymbol{E})$ and dal ${ }^{k n u 6}$ mutant $(\boldsymbol{F})$ embryos with anti-BrdU and anti-BLBP antibodies. $\mathbf{G}-\boldsymbol{I}$, Lateral views of the spinal cord of wt $(\boldsymbol{G})$ and dal ${ }^{k n u 6}$ mutant $(\boldsymbol{H})$ embryos transplanted with $T g($ oligz:egfp) cells, and wt $(\boldsymbol{I})$ embryos transplanted with leo1 M0-injected Tg(olig2:egfp) cells. J, $\boldsymbol{K}$, Quantification of TUNEL ${ }^{+}(\boldsymbol{J})$ and BrdU ${ }^{+}(\boldsymbol{K})$ cells (** $\left.^{*}<0.001\right)$. Scale bar: $20 \mu \mathrm{m}$.

BrdU labeling, immunohistochemistry and in situ RNA hybridization. Dechorionated embryos were labeled with BrdU (Roche) by incubating them for $20 \mathrm{~min}$ on ice in a solution of $10 \mathrm{~mm}$ BrdU and 15\% DMSO in embryo medium (EM) at 3 dpf. The embryos were then placed in EM, incubated for $20 \mathrm{~min}$ at $28.5^{\circ} \mathrm{C}$, and fixed using $4 \%$ paraformaldehyde in PBS. Embryos were processed for immunohistochemistry, treated for $1 \mathrm{~h}$ with $2 \mathrm{M} \mathrm{HCl}$, and then processed for anti-BrdU immunohistochemistry. For immunohistochemistry, we used the following primary antibodies: a mouse anti-BrdU (G3G4, 1:1000, DSHB, IA), a rabbit anti-Sox10 (1: 1000) (Park et al., 2005), a mouse anti-Neurolin (zn-8, 1:1000, DSHB), a rabbit anti-BLBP (1:100, Abcam), rabbit anti-MBP (1:100) (Lyons et al., 2005), and a mouse anti-HuC/D (16A11, 1:20, Invitrogen). Alexa 488-, 568 -conjugated secondary antibodies were used for fluorescence detection (1:500, Invitrogen). In situ RNA hybridization was performed as previously described (Hauptmann and Gerster, 2000), and dm20 RNA probe was used to detect oligodendrocytes (Park et al., 2002).

TUNEL assay. TUNEL assay was performed using In Situ Cell Death Detection Kit (Roche) according to the manufacturer's instructions. TUNEL was done on $10 \mathrm{~mm}$-thick cryosections.

Chemical treatment. Embryos were dechorionated and incubated in EM containing $2.5 \mu \mathrm{M}$ Flavopiridol (FVP, $10 \mathrm{mg} / \mathrm{ml}$; sc-202157, Santa Cruz Biotechnology), $500 \mu \mathrm{g} / \mathrm{ml} \mathrm{5,} \mathrm{6-Dichloro-1-} \beta$-D-ribofuranosylbenzimidazole (DRB, $25 \mathrm{mg} / \mathrm{ml}$; 287891, Calbiochem), $2.5 \mu \mathrm{M}$ Purvalanol A (10 mм; P4484, Sigma) from 24 to $72 \mathrm{hpf}$ or from 24 to $96 \mathrm{hpf}$.

Transplantation. We injected one- to two-cell-stage of $\mathrm{Tg}$ (olig2:egfp) donor embryos with rhodamine-dextran dye alone or together with leol morpholino. Injected embryos were maintained in the dark at $28.5^{\circ} \mathrm{C}$ in embryo medium until sphere stage, and thirty to 40 cells were transplanted from donor embryos into wild-type and dal ${ }^{k n u}$ mutant embryos, respectively.

FACS and $q R T-P C R$. Approximately $3002 \mathrm{dpf} T g$ (sox10:egfp); dal ${ }^{k n u 6}$ embryos (Dutton et al., 2008) were used to isolate $\mathrm{GFP}^{+}$cells by FACS. Cell dissociation and FACS were performed as previously described (Takada and Appel, 2010) using a FACSAriaII (Becton Dickinson). Cells $\left(5 \times 10^{5}-1 \times\right.$ $10^{6}$ ) were isolated and subsequently homogenized in TRIzol solution (Invitrogen) to purify total RNA. Real-time PCR (qRT-PCR) was performed using a LightCycler $\mathrm{R}$ (Roche) in a reaction mixture containing $1 \mu \mathrm{l}$ of PCR-amplified total cDNA as a template, $0.2 \mathrm{M} 5^{\prime}$ and $3^{\prime}$ PCR primer, $0.8 \mu \mathrm{l}$ of $\mathrm{MgCl} 2$, and $1 \mu \mathrm{l}$ of LightCycler R Fast Start DNA Master SYBR Green I (Roche). The following oligonucleotide primers were used for qRT-PCR: $c d k n 1 b$ F (5'-TGATGATCGTCTTGTCGATGT-3'); R (5'-GCTCTTCATG ATCCACCGG-3'); $c d k n 1 c$ F (5'-CAAGAATCCGAGGGAGTCCC-3'); R (5'-GTTCATCCTGCTTCGACTCC- $\left.3^{\prime}\right)$; sox 10 F (5'-GCACCACAATCG ACACAAAC-3'); R (5'-ATCCGGAGTTCAGGAAGGAT-3'); quaking $\mathrm{F}$ (5'-ACATTAAAACCCCCGCAGT-3');R(5'-GTCCTTCCGTACTCGTCC AA-3'); id $2 b$ F (5'-GCTCAGTCTACTGTACAACATGA-3'); R (5' -GCTC CCAGTGATCTGACAGT- $\left.3^{\prime}\right)$; id4 F (5'-GGTCAACTATCAAACATGCG ATG-3'); R (5' -CTGGTCAACACACGTCACCT-3'); sox5 F (5' -TGAGCC CCTACGCCCAGCACAA-3'); R (5' -TGGCTCGTTCTTGATGAGTTCC$\left.3^{\prime}\right)$; sox6 F (5'-GCAGAATCATGTCTTCCAAGC-3'); R (5'-GATTGGCT GGAGCTCCTC-3'); tcf4 F (5'-GGGACGGATAAGGAACTCAGC-3'); R (5'-GTGGCCCGGACTCCATT-3'); $\beta$-actin F (5'-TAGTCATTCCAGAA GCGTTTACC-3'); R (5'-TACAGAGACACCCTGGCTTACAT-3').

\section{Results}

\section{Zebrafish PAF1C is required for oligodendrocyte} differentiation

To screen for mutants with defects in oligodendrocyte development, we used $\operatorname{Tg}$ (olig2:egfp) zebrafish, which express EGFP under the control of the olig2 promoter in the pMN domain of the ventral spinal cord (Shin et al., 2003). As was shown in a previous study, EGFP fluorescence is detected in the motor axon bundles at $2 \mathrm{~d}$ postfertilization (dpf) (Fig. 1A, arrowheads) and in oligodendrocyte lineage cells at $4 \mathrm{dpf}$ in the dorsal spinal cord (Fig. $1 \mathrm{~B}$, bracketed area) of $\mathrm{Tg}$ (olig2:egfp) embryos. In this study, $\mathrm{N}$-ethyl- $\mathrm{N}$-nitrosourea (ENU)-based mutagenesis was performed using $\operatorname{Tg}$ (olig2:egfp) zebrafish and a mutant, dalmuri ${ }^{k n u 6}\left(\right.$ dal $\left.^{k n u \sigma}\right)$, was identified in which olig2-expressing pMN precursors and motor axon bundles were formed normally (Fig. 1C, arrowheads) by $2 \mathrm{dpf}$. However, the pMN precursors failed to generate oligodendrocytes migrated dorsally above the olig2-expressing pMN domain at $4 \mathrm{dpf}$ (Fig. $1 D$, bracketed area), indicating that oligodendrocyte development was impaired in the dal $^{k n u \sigma}$ mutant embryos. 
Positional cloning of the mutated gene in the $d a l^{k n u 6}$ mutant revealed a G to T substitution at nucleotide position 124 in exon 2 of leo1 on chromosome 18. This mutation resulted in a premature stop codon (TGA) at Gly-42 (GGA), generating a truncated protein of 41 aa (Fig. $1 E$ ). Leol is a member of PAF1C, which is known to be a positive regulator of transcription elongation (Jaehning, 2010). Recently, Nguyen et al. (2010) has identified a mutation in another allele of leo1(leo1 $\left.{ }^{\text {LA1186}}\right)$, which exhibited distinct recessive defects in pigment cells and in heart development (Nguyen et al., 2010). The $d a l^{k n u 6}$ mutant identified in the current study showed the same phenotype as leo1 $1^{\text {LA1186 }}$, including defects in heart and pigment development (Fig. $1 F$ ). Knockdown of leo1 with a targeted morpholino phenocopied the dal ${ }^{k n u 6}$ mutant (data not shown) while ectopic expression of normal leo $1 \mathrm{mRNA}$ rescued the dal ${ }^{k n u}$ mutant phenotype (Fig. $1 F$ ).

Consistent with normal motor axon bundle formation (Fig. 1C), the dal ${ }^{k n u 6}$ mutant embryo generated the usual number of Neurolin ${ }^{+}$motor neurons (Fig. $1 G, H)$. Labeling of the spinal cord sections with anti-Sox10 and anti-Hu antibodies, which mark oligodendrocyte lineage cells and neurons, respectively, showed that the dal ${ }^{k n u \sigma}$ mutant embryo generated the normal number of OPCs and neurons in the gray matter of the spinal cord at $2 \mathrm{dpf}$, similar to the wild-type embryo (Fig. $1 \mathrm{~J}, \mathrm{~K}$, arrowheads indicate OPCs). However, compared with wild-type, in which OPCs migrate and differentiate into mature oligodendrocytes in the white matter of spinal cord at $4 \mathrm{dpf}$ (Fig. $1 \mathrm{M}$, arrowheads), OPCs had disappeared from the $d a l^{k n u 6}$ mutant embryo by this stage of development (Fig. 1N). Consistent with these phenotypes, whole-mount in situ RNA hybridization with $p l p / d m 20$, a mature oligodendrocyte marker, revealed that there were no $p l p / d m 20^{+}$mature oligodendrocytes in the spinal cord of the $d a l^{k n u}$ mutant at $4 \mathrm{dpf}$ (Fig. $1 P, Q$ ), indicating that leol deficiency causes impaired oligodendrocyte differentiation. Knockdown of $c d c 73$, another member of PAF1C, by injection of a $c d c 73$ morpholino, also caused impaired oligodendrocyte differentiation that was similar to that seen in the dal ${ }^{k n u 6}$ mutant embryo (Fig. $1 I, L, O, R$ ), suggesting that PAF1C function is required for the differentiation of OPCs into myelinating oligodendrocytes in the spinal cord of zebrafish embryos.

Cell autonomous functioning of Leo1 is required for OPC differentiation and neural precursor cell cycle regulation Since OPCs disappeared without differentiating into myelinating oligodendrocytes in dal ${ }^{k n u 6}$ mutant embryos, we investigated
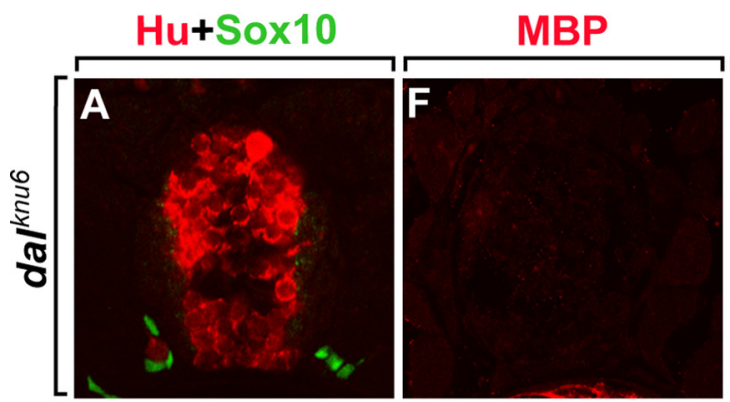

\section{BrdU+Sox10}
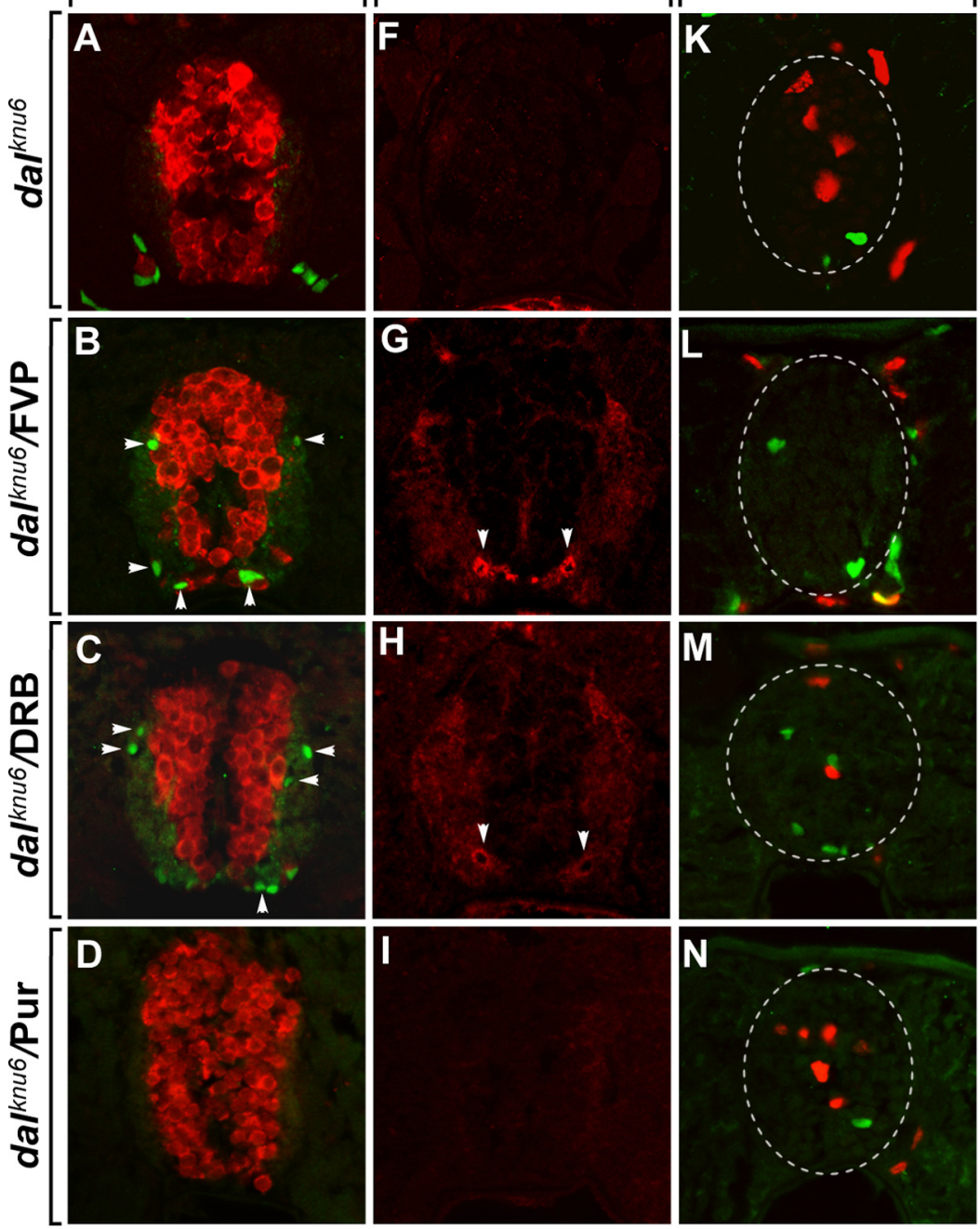

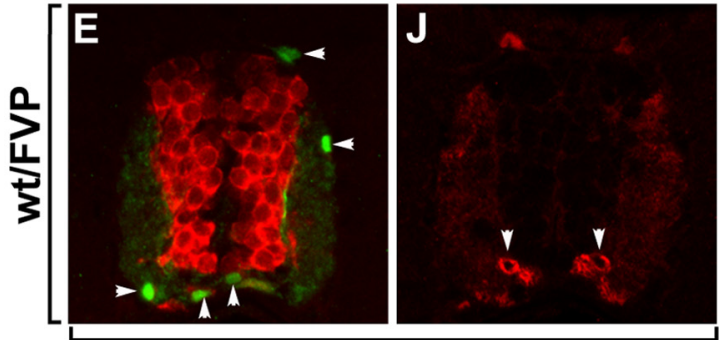

$4 \mathrm{dpf}$

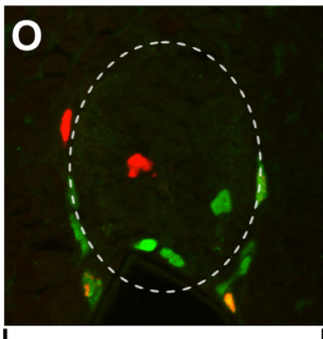

$3 \mathrm{dpf}$
Figure 3. Inhibition of p-TEFb function rescues impaired oligodendrocyte differentiation in da ${ }^{k n u 6}$ mutant embryos. Transverse sections of spinal cord from wild-type and $d a f^{k n u 6}$ mutant embryos treated with chemical inhibitors, oriented dorsal to the top. Chemical inhibitors are indicated on each panel. Embryos were incubated in chemicals from 24 to $96 \mathrm{hpf}(\boldsymbol{B}-\boldsymbol{E}, \boldsymbol{G}-\boldsymbol{J})$ and from 24 to $72 \mathrm{hpf}(\boldsymbol{L}-\mathbf{0})$. Control embryos were incubated in $\operatorname{EM}(\boldsymbol{A}, \boldsymbol{F}, \boldsymbol{K})$. Antibodies and stages are indicated on each panel. Arrowheads indicate Sox10 ${ }^{+}$myelinating oligodendrocytes in the white matter $(\boldsymbol{B}-\boldsymbol{E})$, and myelin processes labeled with an anti-MBP antibody $(\boldsymbol{G}-\boldsymbol{J})$. Dashed circle outlines spinal cord $(\boldsymbol{K}-\mathbf{0})$. Scale bar: $20 \mu \mathrm{m}$.

next whether OPCs were eliminated by apoptosis. To visualize mature oligodendrocytes in vivo, nkx2.2a:megfp and olig2:dsred transgenes were introduced into the $\mathrm{dal}^{k n u 6}$ mutant. These transgenes drive the membrane targeting of GFP (mGFP) and Dsred proteins in myelinating oligodendrocytes under control of the $n k x 2.2 a$ and olig2 promoters, respectively (Kucenas et al., 2008). At $3 \mathrm{dpf}$, the $\mathrm{mGFP}^{+} /$Dsred $^{+}$myelinating oligodendrocytes $^{-}$ showed numerous processes in the dorsal spinal cord of the wildtype $\operatorname{Tg}(n k x 2.2 a: m g f p ; o l i g 2: d s r e d)$ embryo (Fig. $2 A$, arrowheads), 
while $\mathrm{mGFP}^{+} /$Dsred $^{+}$myelinating oligodendrocytes were rarely observed and showed abnormal morphology in the $\operatorname{Tg}(n k x 2.2 a: m g f p:: o l i g 2: d s r e d) ; d a l^{k n u 6}$ mutant embryo (Fig. $2 B$, arrowheads), suggesting that they had been eliminated by cell death. Consistent with this result, $d a l^{k n u 6}$ mutant embryos had increased numbers of TUNEL ${ }^{+}$cells compared with the wildtype embryo, indicating that OPCs undergo apoptosis (Fig. $2 C, D, J)$.

Interestingly, dal ${ }^{k n u 6}$ mutant embryos exhibited normal proliferation during neurogenesis at $24 \mathrm{hpf}$ (data not shown), but showed a dramatic increase in the number of $\mathrm{BLBP}^{+} / \mathrm{BrdU}^{+}$ proliferating radial glial precursors after neurogenesis was complete at $2 \mathrm{dpf}$, indicating that the Leo 1 deficiency causes defects in cell-cycle regulation in neural precursors (Fig. $2 E, F, K$ ). Since OPCs are still proliferating cells and would normally stop cycling before differentiating into myelinating oligodendrocytes, these data suggest that impaired OPC differentiation might be partly due to a failure in OPC cell cycle regulation.

To test whether Leol functions in a cell autonomous manner, rhodamine-dextran dye was injected into the one-cell stage of wild-type $T g$ (olig2:EGFP) embryos and cells were transplanted to nontransgenic wild-type and dal ${ }^{k n u 6}$ mutant embryos at the blastula stage. In the wild-type and dal ${ }^{k n u 6}$ mutant host embryos, transplanted $\operatorname{Tg}($ olig2:EGFP) cells successfully differentiated into myelinating oligodendrocytes, which showed the typical morphology with multiple processes (Fig. $2 G, H$ ). However, transplanted leo1-deficient cells from the $T g($ olig2:EGFP) embryos injected with leo1 morpholino together with rhodamine-dextran dye failed to produce mature oligodendrocytes in the wild-type embryos, indicating that the dal ${ }^{k n u}$ mutant phenotype is caused by the cell autonomous function of Leo1 (Fig. 2I). Altogether, these data indicate that the cell-autonomous function of Leol is required for the regulation of neural precursor proliferation and oligodendrocyte differentiation in the spinal cord of zebrafish embryos.

\section{PAF1C and p-TEFb regulate oligodendrocyte differentiation antagonistically}

A previous study demonstrated that antagonistic regulation of PAF1C and p-TEFb is required for erythroid-specific gene expression by regulating Pol II pausing mechanism (Bai et al., 2010). Therefore, we hypothesized that a functional antagonism between PAF1C and p-TEFb may be required for the regulation of oligodendrocyte development. To test this idea, we used flavopiridol (FVP), a chemical inhibitor of CDK9, to block p-TEFb function (Chao and Price, 2001; Bai et al., 2010). Interestingly, FVP treatment before OPC specification at $24 \mathrm{hpf}$ rescued most of the mutant phenotype in the spinal cord of dal ${ }^{k n u}$ mutant embryos. Sox $10^{+}$myelinating oligodendrocytes showing numerous processes were observed in the white matter of the spinal cord in dal ${ }^{k n u 6}$ mutant embryos treated with FVP (Fig. 3B, arrowheads). Consistent with this, labeling with an anti-Myelin Basic Protein (MBP) antibody, which labels the myelin of mature oligodendrocytes (Lyons et al., 2005), revealed recovery of myelination in the spinal cord of dal ${ }^{k n u}$ mutant embryos (Fig. 3G, arrowheads). Normal proliferation of spinal precursors in the dal ${ }^{k n u 6}$ mutant embryos was also restored following FVP treatment (Fig. 3L). Similarly, treatment with 5, 6-Dichloro-1- $\beta$-Dribofuranosylbenzimidazole (DRB), which blocks CTD kinase activity and transcriptional function of p-TEFb (Dubois et al., 1994), also rescued impaired oligodendrocyte differentiation (Fig. $3 \mathrm{C}, \mathrm{H}$ ) and precursor proliferation (Fig. $3 \mathrm{M}$ ) in $\mathrm{dal}^{\mathrm{knu}} \mathrm{mu}$ tant embryos. However, dal ${ }^{k n u}$ mutant embryos treated with

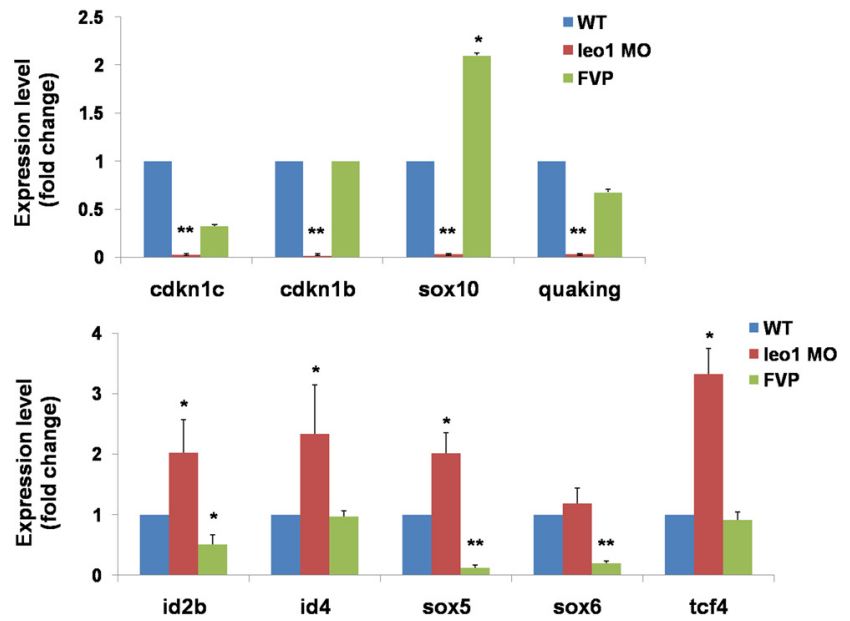

Figure 4. PAF1C and p-TEFb antagonistically regulate gene expression in OPCs. Real-time RT-PCR analyses to compare the expression of OPC genes in $\mathrm{GFP}^{+}$cells isolated from wild-type (wt), leo1 morphants and FVP-treated leo 1 morphants. Data are shown as average fold change relative to the wild-type control and normalized to $\beta$-actin expression. The results presented as mean \pm SD from three independent experiments $\left({ }^{*} p<0.05\right.$; $\left.{ }^{* *} p<0.001\right)$.

Purvalanol A, which is a protein kinase inhibitor with high selectivity for cell cycle-associated CDKs, including CDK1, CDK2 and CDK4 (Villerbu et al., 2002), failed to rescue impaired oligodendrocyte differentiation and precursor proliferation (Fig. $3 D, I, N)$. Interestingly, oligodendrocyte differentiation and precursor proliferation are not affected in FVP-treated wild-type embryos, indicating that loss of $\mathrm{p}$-TEFb function alone does not affect oligodendrocyte differentiation (Fig. $3 E-O$ ). Next, we demonstrated that treating $c d c 73$ morpholino-injected embryos (morphants) with FVP also rescued impaired oligodendrocyte differentiation (data not shown). Together, these data indicate that inhibition of p-TEFb function rescues impaired oligodendrocyte differentiation in dal ${ }^{k n u}$ mutant embryos, and that PAF1C and $\mathrm{p}-\mathrm{TEFb}$ antagonistically regulate oligodendrocyte differentiation in the CNS.

Finally, the regulation of gene expression by Leo 1 and $\mathrm{p}-\mathrm{TEFb}$ was examined in OPCs. A morpholino targeted to leol was injected into sox10:EGFP transgenic embryos, in which oligodendrocyte lineage cells were labeled with EGFP (Dutton et al., 2008). $\mathrm{EGFP}^{+}$OPCs were isolated by fluorescent activated cell sorting (FACS) before undergoing apoptosis. Quantitative Reverse Transcription PCR (qRT-PCR) was then performed to compare the expression levels of the selected genes in wild-type and leo 1 morphants, and leo1 morphants treated with FVP. We first analyzed expressions of $c d k n 1 b$ (Casaccia-Bonnefil et al., 1997), $c d k n 1 c$ (Park et al., 2005), quaking (Larocque and Richard, 2005) and sox10 (Stolt et al., 2002), which are required for the differentiation of OPCs into myelinating oligodendrocytes. As shown in Figure 4, qRT-PCR analysis showed that expressions of selected genes were downregulated in leo1-deficient OPCs, and their expressions were rescued upon FVP treatment (Fig. 4). We next analyzed the expression of negative regulators of myelination, including $i d 2 b$, $i d 4$, sox 5 , sox6, and $t c f 4$, which are transcription factors required for the maintenance of OPCs in an undifferentiated state and to repress myelin gene expression (Emery, 2010). Interestingly, expression of these genes was significantly upregulated in the leo1-deficient OPCs and was rescued upon FVP treatment (Fig. 4); this suggests that a failure in the repression of negative regulators of myelination due to the loss of PAF1C causes downregulation of the genes required for oligodendrocyte 
differentiation in dal ${ }^{k n u 6}$ mutant embryos. FVP treatment in leo1deficient OPCs unexpectedly downregulated the expression of sox 5 and sox6 to below the level observed in wild-type, and upregulated sox10 expression above wild-type levels. Given that Sox 5 and Sox 6 both regulate oligodendrocyte development by interfering with the function of Sox9 and Sox10, dramatic upregulation of sox10 appears to be related to downregulation of sox 5 and sox6. Altogether, these data show that PAF1C plays a negative role, and $\mathrm{p}-\mathrm{TEFb}$ a positive role, in the transcriptional regulation of negative regulators of myelination, and functional antagonism between PAF1C and p-TEFb is required for the regulation of oligodendrocyte differentiation.

\section{Discussion}

\section{PAF1C is required for oligodendrocyte development}

Despite numerous biochemical and genetic studies, the biological roles of PAF1C in multicellular organisms are largely unknown. In the present study, we report that the leo1 mutation in dal ${ }^{k n u}$ mutant embryos causes impaired oligodendrocyte differentiation in the CNS in addition to other known defects. Cdc73 morphants also showed similar CNS defects to those in dal ${ }^{k n u 6}$ mutant embryos, indicating that impaired oligodendrocyte development is caused by a defect in PAF1C function. Interestingly, even though leo 1 is broadly expressed in the CNS, including neurons, neuronal differentiation was normal in $d a l^{k n u 6}$ mutants, suggesting a specific role for PAF1C in oligodendrocyte development. However, the mechanisms responsible for the differential roles of PAF1C in specific tissues are largely unknown. Previously, it was shown that PAF1C interacts with the Wnt and Hedgehog signaling pathways in mammalian cells (Mosimann et al., 2006, 2009). Therefore, the specific defect in oligodendrocyte development in the CNS of $d a l^{k n u 6}$ mutants could be caused by the loss of interaction with particular genes and signaling pathways important for oligodendrocyte development.

\section{Antagonistic regulation of oligodendrocyte differentiation by PAF1C and p-TEFb}

Cell fate specification during development depends upon the precise regulation of tissue-specific gene expression programs. After transcription initiation with tissue-specific transcription factors, promoter-proximal pausing of RNA Pol II is prevalent at developmentally regulated promoters, suggesting that "pausing" mechanisms might facilitate precise, synchronous expression of developmental genes (Muse et al., 2007; Zeitlinger et al., 2007; Core et al., 2008; Price, 2008). Previous genome-wide analysis of Pol II pausing in Drosophila embryos revealed two nonexclusive developmental functions of Pol II pausing; it serves as a mechanism for the active transcriptional repression of some genes, and as a mechanism for the rapid induction of other genes at later stages of embryogenesis (Zeitlinger et al., 2007). A recent study shows that the Pol II pausing mechanism mediated by functional antagonism between PAF1C and $\mathrm{p}-\mathrm{TEFb}$, which play negative and positive roles in transcriptional activation, respectively, is required for the erythroid gene expression (Bai et al., 2010). In this case, Pol II pausing seems to serve as a mechanism that allows rapid and synchronized activation of erythroid genes. Consistent with this, deficiency of the negative regulator for transcription, PAF1C, in the sunrise mutant did not affect erythroid gene expression because of the existence of the positive transcriptional activator, $\mathrm{p}$-TEFb. However, $\mathrm{p}$-TEFb deficiency in the moonshine mutant causes downregulation of erythroid gene expression, which is rescued by PAF1C deficiency (Bai et al., 2010). Similar to erythropoiesis, our current data demonstrate that an- tagonistic regulation of PAF1C and p-TEFb is required for the differentiation of OPCs into myelinating oligodendrocytes, suggesting the existence of a Pol II pausing mechanism mediated by PAF1C and p-TEFb. However, unlike erythropoiesis, deficiency in the positive regulator of transcription, $\mathrm{p}$-TEFb, in FVP-treated wild-type embryos does not affect oligodendrocyte differentiation, presumably because the negative regulator of transcription, PAF1C, might successfully downregulate negative regulators of myelination (Fig. 3E,J). However, PAF1C deficiency in dal ${ }^{k n u 6}$ mutant embryos resulted in upregulation of negative regulators of myelination, and was rescued by $\mathrm{p}-\mathrm{TEFb}$ deficiency upon FVP treatment (Fig. 4). These data suggest that the Pol II pausing mechanism is required for the repression of negative regulators of myelination to induce oligodendrocyte differentiation. Due to technical limitations, we did not find direct evidence of a role for the Pol II pausing mechanism in the regulation of oligodendrocyte differentiation, but our data show that antagonistic regulation of gene expression by the transcription elongation factors, PAF1C and p-TEFb, is complex, and occurs widely during development.

\section{References}

Bai X, Kim J, Yang Z, Jurynec MJ, Akie TE, Lee J, LeBlanc J, Sessa A, Jiang H, DiBiase A, Zhou Y, Grunwald DJ, Lin S, Cantor AB, Orkin SH, Zon LI (2010) TIFlgamma controls erythroid cell fate by regulating transcription elongation. Cell 142:133-143.

Casaccia-Bonnefil P, Tikoo R, Kiyokawa H, Friedrich V Jr, Chao MV, Koff A (1997) Oligodendrocyte precursor differentiation is perturbed in the absence of the cyclin-dependent kinase inhibitor p27Kip1. Genes Dev 11:2335-2346.

Chao SH, Price DH (2001) Flavopiridol inactivates P-TEFb and blocks most RNA polymerase II transcription in vivo. J Biol Chem 276:31793-31799.

Chiba K, Yamamoto J, Yamaguchi Y, Handa H (2010) Promoter-proximal pausing and its release: molecular mechanisms and physiological functions. Exp Cell Res 316:2723-2730.

Core LJ, Waterfall JJ, Lis JT (2008) Nascent RNA sequencing reveals widespread pausing and divergent initiation at human promoters. Science 322:1845-1848.

Dubois MF, Nguyen VT, Bellier S, Bensaude O (1994) Inhibitors of transcription such as 5,6-dichloro-1-beta-D-ribofuranosylbenzimidazole and isoquinoline sulfonamide derivatives (H-8 and H-7) promote dephosphorylation of the carboxyl-terminal domain of RNA polymerase II largest subunit. J Biol Chem 269:13331-13336.

Dutton JR, Antonellis A, Carney TJ, Rodrigues FS, Pavan WJ, Ward A, Kelsh RN (2008) An evolutionarily conserved intronic region controls the spatiotemporal expression of the transcription factor Sox10. BMC Dev Biol 8:105.

Emery B (2010) Regulation of oligodendrocyte differentiation and myelination. Science 330:779-782.

Hager GL, McNally JG, Misteli T (2009) Transcription dynamics. Mol Cell 35:741-753

Hauptmann G, Gerster T (2000) Multicolor whole-mount in situ hybridization. Methods Mol Biol 137:139-148.

Jaehning JA (2010) The Pafl complex: platform or player in RNA polymerase II transcription? Biochim Biophys Acta 1799:379-388.

Kucenas S, Takada N, Park HC, Woodruff E, Broadie K, Appel B (2008) CNS-derived glia ensheath peripheral nerves and mediate motor root development. Nat Neurosci 11:143-151.

Larocque D, Richard S (2005) QUAKING KH domain proteins as regulators of glial cell fate and myelination. RNA Biol 2:37-40.

Lyons DA, Pogoda HM, Voas MG, Woods IG, Diamond B, Nix R, Arana N, Jacobs J, Talbot WS (2005) erbb3 and erbb2 are essential for schwann cell migration and myelination in zebrafish. Curr Biol 15:513-524.

Mosimann C, Hausmann G, Basler K (2006) Parafibromin/Hyrax activates $\mathrm{Wnt} / \mathrm{Wg}$ target gene transcription by direct association with beta-catenin/ Armadillo. Cell 125:327-341.

Mosimann C, Hausmann G, Basler K (2009) The role of Parafibromin/Hyrax as a nuclearGli/Ci-interacting protein in Hedgehog target gene control. Mech Dev 126:394-405. 
Muse GW, Gilchrist DA, Nechaev S, Shah R, Parker JS, Grissom SF, Zeitlinger J, Adelman K (2007) RNA polymerase is poised for activation across the genome. Nat Genet 39:1507-1511.

Nguyen CT, Langenbacher A, Hsieh M, Chen JN (2010) The PAF1 complex component Leol is essential for cardiac and neural crest development in zebrafish. Dev Biol 341:167-175.

Park HC, Boyce J, Shin J, Appel B (2005) Oligodendrocyte specification in zebrafish requires notch-regulated cyclin-dependent kinase inhibitor function. J Neurosci 25:6836-6844.

Park HC, Mehta A, Richardson JS, Appel B (2002) olig2 is required for zebrafish primary motor neuron and oligodendrocyte development. Dev Biol 248:356-368.

Price DH (2008) Poised polymerases: on your mark ... get set ... go! Mol Cell 30:7-10.

Rowitch DH (2004) Glial specification in the vertebrate neural tube. Nat Rev Neurosci 5:409-419.
Shin J, Park HC, Topczewska JM, Mawdsley DJ, Appel B (2003) Neural cell fate analysis in zebrafish using olig2 BAC transgenics. Methods Cell Sci 25:7-14.

Stolt CC, Rehberg S, Ader M, Lommes P, Riethmacher D, Schachner M, Bartsch U, Wegner M (2002) Terminal differentiation of myelinforming oligodendrocytes depends on the transcription factor Sox10. Genes Dev 16:165-170.

Takada N, Appel B (2010) Identification of genes expressed by zebrafish oligodendrocytes using a differential microarray screen. Dev Dyn 239:2041-2047.

Villerbu N, Gaben AM, Redeuilh G, Mester J (2002) Cellular effects of Purvalanol A: a specific inhibitor of cyclin-dependent kinase activities. Int J Cancer 97:761-769.

Zeitlinger J, Stark A, Kellis M, Hong JW, Nechaev S, Adelman K, Levine M, Young RA (2007) RNA polymerase stalling at developmental control genes in the Drosophila melanogaster embryo. Nat Genet 39:1512-1516. 